\title{
A Fuzzy Logic Approach for Indoor Mobile Robot Navigation using UKF and Customized RFID Communication
}

\author{
M. Suruz Miah and Wail Gueaieb \\ School of Information Technology and Engineering \\ University of Ottawa, Ottawa, Ontario, Canada \\ Email: suruz·miah@uOttawa·ca,wgueaieb@site·uOttawa·ca
}

\begin{abstract}
Most common techniques pertaining to the problem of mobile robot navigation are based on the use of multiple sensory data fusion and of excessive number of reference Radio Frequency (RF) stations. However, spatial layout, high navigation accuracy, and cost problems limit the applicability of those techniques in many modern robotic applications. The current manuscript outlines a novel computationally inexpensive indoor mobile robot navigation system with an intelligent processing of received signal strength (RSS) measurements of a customized Radio Frequency IDentification (RFID) system. The high navigation accuracy is achieved by incorporating a conventional stochastic Uncented Kalman Filter (UKF), which integrates RSS measurements from a customized RFID system and sensory data from the robot's wheel encoder. The workspace is defined by placing a number of RFID tags at 3-D locations. The orthogonal 2-D projection points of those tags on the ground define the target points where the robot is supposed to reach. The customized RFID system is simulated using a comprehensive electromagnetic commercial software, FEKO. The validity and suitability of the proposed navigation system are demonstrated by conducting extensive computer simulations in order to provide high-performance level in terms of accuracy and scalability.
\end{abstract}

Keywords: Customized RFID reader, fuzzy logic controller, mobile robot navigation, perception, received signal strength, UKF.

\section{Introduction}

RFID technology has been extensively deployed recently in the field of robotics thanks to its simplicity, compact size, cheap price, and flexibility [1]. The navigation problem of a mobile robot has been traditionally solved by several approaches suggested in the literature. One of the most popular techniques is the RSS-based navigation/localization using RFID technology where the line-of-sight (LoS) distance (or range) between an RFID tag and a reader is computed by Friss transmission formula [2]. In general, RSS is a feature of choice for estimating range in wireless networks because it can be obtained relatively effortlessly and inexpensively (i.e., no extra hardware is required) [3] which makes it good candidate to solve a localization problem. However, it has lately been discovered that achieving accurate mapping of RSS information to range is almost 
impossible in real-world reverberant environment [4], depleting the advantages listed above.

Hence, our current work contributes to the development of a mobile robot navigation system where accurate mapping of RSS measurements to LoS distance is not absolutely necessary. The robot is assumed to be equipped with a customized RFID reader and the environment is defined by placing a set of RFID tags at 3-D points whose orthogonal projection points on the ground define the target points to be reached by the robot. The RFID system architecture is discussed later in the paper. The majesty of this work is that the direction of the target point is determined using RSS values which are provided by the customized RFID reader. The robot then updates its orientation towards the target point followed by a set of control actions to reach that point which are determined by the processing of RSS measurements using a fuzzy logic controller (FLC). The robot's position accuracy is refined through a conventional stochastic filter, UKF.

The rest of the manuscript is outlined as follows. Some of the most commonly used RFID-based robot navigation systems are presented in section 2. Section 3 illustrates the proposed navigation framework. The robot's noisy positions and orientations are filtered using the UKF which is described in section 4 The performance of the proposed navigation system is then evaluated numerical experiments reported in section 5 . Finally, section 6 concludes with some key advantages of the current research in real-life.

\section{Related Study}

Due to the recent advancements in RFID technology, and its applications in the field of robotics, localization systems have been used to deliver instantaneous position information in an indoor and/or outdoor environment. A large body of research has considered localizing a mobile robot using the emerging RFID technology. Since an RFID system can recognize objects at high-speed and send data within various distances, RFID technology has been gaining popularity within the robot community [5]. Hahnel et al. studied to improve robot localization with a pair of RFID antennas [6]. They presented a probabilistic measurement model for RFID readers that allow them to accurately localize the RFID tags in the environment.

In addition, robot's position estimation techniques can be classified as range-based and bearing-based [4]. The main idea behind range-based techniques is to trilaterate the robot's position using some known reference points and the estimated distances at those points in the environment. Distances can be estimated from either RSS measurements or time-based methods. A small subset of such works have explored the use of Time of Flight (ToF) [7] or Time Difference of Arrival (TDoA) measurements [8]. On the other hand, bearing-based schemes use the direction of arrival (DoA) of a target. However, these schemes require multiple range sensors in order to be better suited for mobile robot applications [4].

Despite the significant contributions of RFID systems and RSS measurements stated in the literature, the navigation problem of a mobile robot still faces technical challenges. For instance, the performance of RSS-based localization and/or navigation systems highly degrades in a reverberant environment and such systems are not applicable where approximating RSS measurements to LoS distance with a high precision is 
required. As such, the current work is devoted to the development of an RSS-based navigation system which might be a promising alternative to the existing robot navigation systems to date.It is noteworthy to mention that approximating RSS measurements to LoS distance, which most of the RSS-based navigation systems depend on, is not absolutely necessary in the proposed navigation system. To the best of the authors knowledge, this is the first attempt of finding direction using RSS measurements of a customized RFID system for the purpose of robot navigation in an indoor environment.

\section{Proposed Navigation System}

A high level setup of the proposed navigation system with a customized RFID reader mounted on a mobile robot is depicted in Fig. 1. The four RFID tags, Tag1, Tag2, Tag3, and Tag4, are attached to the 3-D points of the ceiling of an indoor office environment, for instance. In this configuration, the robot's target points, p1, p2, p3, and p4, are the orthogonal projection points of those tags on the ground. The mobile robot is pre-programed with these four tag IDs (a set of RFID tag IDs in general case) and is supposed to reach $\mathrm{p} 1$, then $\mathrm{p} 2$, after that $\mathrm{p} 3$, and finally $\mathrm{p} 4$. For it to do so, the robot continuously reads tag IDs using the customized RFID reader, and extracts and processes RSS values of the signal coming from the current target tag at a particular time instant. The communication between the tags and the reader is performed through the multiple receiving antennas mounted on the reader.

The current work is mainly composed of three components: a mobile robot, a customized RFID system, and a guiding principle. These components rely on the fact that an accurate data processing and control actions are performed by the robot while navigating in its workspace. In the following, we describe these components in detail.

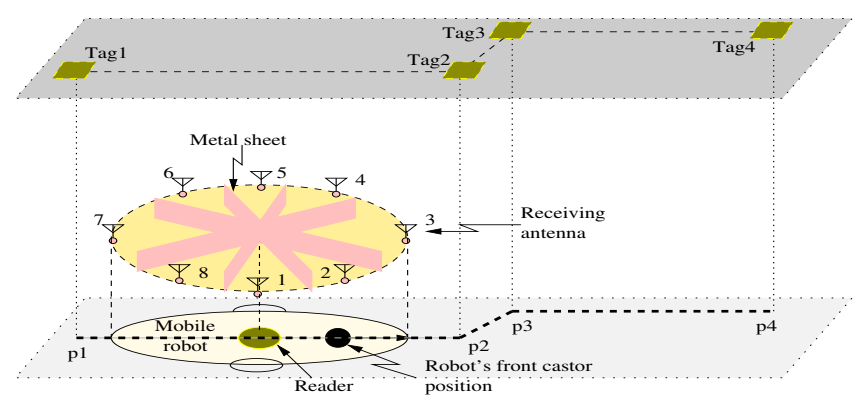

Fig. 1. High level system architecture of the proposed navigation system.

\subsection{Robot Model}

The current work deploys a differential drive mobile robot. To derive its kinematic model, let us consider $(x, y)$ and $\gamma$ being the position and the heading angle of a robot 
with respect to a ground-fixed inertial reference frame X-Y. The rotational velocities of the robot's left and right wheels are characterized by the sole (scaler) axis angular velocities $\omega_{L}$ and $\omega_{R}$, respectively; under the assumption that the front castor wheel is passive and simply provides necessary support for its balance. The robot's position is the midpoint of the wheelbase of length $l_{b}$ connecting the two lateral wheels along their axis [9].

The discrete-time equivalent of the robot's kinematic model at time $k$ can be derived as

$$
q(k)=\left[\begin{array}{l}
x(k) \\
y(k) \\
\gamma(k)
\end{array}\right]=q(k-1)+T \frac{r}{2}\left[\begin{array}{cc}
\cos \gamma(k-1) & \cos \gamma(k-1) \\
\sin \gamma(k-1) & \sin \gamma(k-1) \\
\frac{2}{l_{b}} & -\frac{2}{l_{b}}
\end{array}\right]\left[\begin{array}{l}
\omega_{R}(k-1) \\
\omega_{L}(k-1)
\end{array}\right]
$$

where $r$ is the radius of each wheel, $T$ is the sampling time in seconds, $q(k) \in \mathbb{R}^{3}$ represents the robot's current pose (position and orientation), and $\left[\omega_{R}(k) \omega_{L}(k)\right]^{T}$ is the control input $u_{k}$ for its actuator. It is worth to mention, however, that the low sampling frequency and high velocities of the robot can be a significant source of odometric error. We employ the FLC and the UKF to resolve odometric problem during the robot's navigation.

\subsection{Customized RFID System}

Most of the commercially available RFID systems on the market provide only static information to its data processing unit which limits its applicability in many real-world proximity-based applications. As such, we pioneer the current work with a customized RFID reader that consists of eight receiving antennas [10]. Note that the tag architecture of the customized RFID system remains the same as that of a typical commercial RFID system. As can be seen in Fig. 11, we customize an RFID reader by designing an antenna array system where eight antennas are placed at fixed angular positions, $\delta_{i}$ with $i=1, \ldots, 8$, of a circle of radius $r_{a}$ centered on a horizontal rectangular metal plate. Note that this metal plate (known as ground plane) of dimension $l \times l$ needs to be mounted on the RFID reader which acts as an RF front-end. As noted in the literature, RF signals can easily be distorted due to reverberation, and therefore the error in RSS measurements drastically increases in reverberant environment. As a result, each receiving antenna on the customized RFID reader is spatially separated from the other antennas by vertical rectangular metal shields of dimension $l \times h_{p}$. The vertical metal shield also reflects the fact that antennas' RSS values of the signal coming from a tag can easily be distinguished from each other.

The receiving antennas are interfaced with the reader's back-end through a 8-to-1 multiplexer and an RSS power detector in addition to its existing analog and digital front-end interface circuits for receiving static binary IDs from RFID tags. The RFID reader can extract RSS values from eight receiving antennas through the multiplexer using time-division multiple-access (TDMA) technique. The eight RSS values along with the current tag ID are then simply passed to the robot's central processing unit for guiding itself in the pre-specified workspace. 


\subsection{Guiding Principle}

The purpose of this section is to compute the direction of the current target RFID tag (DOCT) and then navigate to reach the 2-D point of that tag.The robot mainly remains at two stages: rotation and navigation, while reaching a set of target points in the workspace. Initially (source state), the robot starts its actuators to rotate itself towards the current target point (rotation stage) based on its reader's RSS measurements of the target tag. Once the current orientation of the robot is updated, it passes the control to the navigation stage and remains in this state by generating appropriate control actions until the target point is reached. If this target point is not the final point in the set of pre-defined target points, then the robot goes back to the rotation stage to update its current heading to the next target point. This process continues until it reaches the final destination point (destination state). In the following, we illustrate how the two main stages can be implemented.

Tuning Robot's Direction Suppose that $\bar{m}_{i}(k)$ is the (noisy) RSS value received by antenna $i$ at time $k, i=1, \ldots, 8$, where $m_{i}(k)$ is the ideal value of that signal (without reverberation nor noise). In other words, $\bar{m}_{i}(k)=m_{i}(k) G\left(\delta_{i}\right)+v_{i}(k)$, where $v_{i}(k)$ is the noise associated with the RSS value received at antenna $i$ and $G\left(\delta_{i}\right)$ is the gain of the antenna in the direction of $\delta_{i}$. A preliminary study has been conducted to ensure that reverberated noisy signal can be mostly filtered out by a simple moving average filter, which is what we used in this work.

The DOCT is approximated by identifying the antenna with the maximum RSS value. That is,

$$
\text { DOCT }=\underset{\delta_{i}, 1 \leq i \leq 8}{\arg \max }\left(\bar{m}_{i}(k)\right) .
$$

Note that the DOCT can be approximated more precisely by comparing the differences between the RSS values of various antenna combinations, i.e., $\bar{m}_{i}(k)-\bar{m}_{j}(k)$, with $i, j=1$ to 8 and $i \neq j$. The robot then updates its current orientation based on the DOCT.

Reaching Target Points Once the direction of the current target on the robot's desired path is detected, it rotates itself towards the target tag as described earlier. Its mission is then to reach the target point which is the tag's orthogonal projection point on the ground. For it to do so, a fuzzy logic controller is employed for generating adequate control actions which are based on stochastic RSS measurements provided by the reader.

Fuzzy logic control is of a paramount importance in many control problems due to its appropriateness in various complex or ill-defined systems. It uses a set of wellstructured if-then rules to represent system's input-output relationships as opposed to complex mathematical models defining the system. Fig.2 2 represents the controller used by the mobile robot. The perception block of the controller aggregates RSS values from eight receiving antennas. However, RSS values from the antenna 2 and 4 are passed to the preprocessing block of the controller for it to reflect that RSS values of these two antennas are sufficient to control speeds of the robot's right and left wheels, respectively. The differential input of the FLC, $\Delta \bar{m}_{24}$, is defined as $\Delta \bar{m}_{24}=\bar{m}_{2}-\bar{m}_{4}$. 
The normalized values of the input $\Delta \bar{m}_{24} \in[-1200,1200] \mathrm{nW}$, are fed to the FLC for producing normalized left and right wheel speeds, $\omega_{L n}$ and $\omega_{R n}$, respectively. The post-processing block is used to scale the normalized speeds to engineering units. In this work, it contains the output speed gain that is tuned to best fit to the wheel actuators. Hence, the actual speeds of both wheels generated by the post-processing block are $\omega_{R}$ and $\omega_{L}$ which are directly applied to the robot's actuators to obtain its new pose using (1).

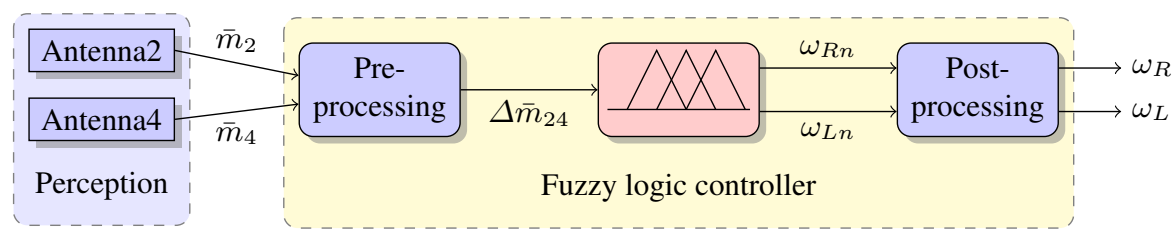

Fig. 2. Robot's speed control mechanism.

\section{Pose Estimation Using UKF}

Although Extended Kalman Filter (EKF) is a widely used state estimation algorithm, it has several drawbacks that can easily be resolved using the Uncented Kalman Filter, UKF. The main advantages of the UKF over the EKF can be sought in [11]. The UKF used in the current work fuses the data from the wheel encoders and RSS measurements in order to better estimate the robot position. It deterministically extracts so-called sigma points from the Gaussian statistics and passes them through the nonlinear model defined in (1).

The UKF localization approach for the robot to approximate its position recursively can be sought in [12]. It integrates the RSS measurements of the RFID reader with the previous pose $q(k-1)$ and the error covariance matrix $P(k-1)$ to estimate its pose $q(k)$ and covariance $P(k)$ at time $k$.

\section{Simulation Results}

In this section, we evaluate the performance of the proposed navigation system using a circular shaped differential drive virtual mobile robot with a wheel base of $30 \mathrm{~cm}$ and a radius $5 \mathrm{~cm}$ of each wheel. The maximum output speed gain of the robot's actuator is $1.5 \mathrm{rad} / \mathrm{s}$. The standard deviation of the left and right wheel rotational speeds are chosen as $0.1 \mathrm{rad} / \mathrm{s}$; and that of the measurement noise associated with the robot's position $(x, y)$ is $0.2 \mathrm{~m}$ and the robot's orientation $\gamma$ is $0.1 \mathrm{rad}$, respectively. The configuration of the proposed RFID reader antenna system using the commercial simulation software FEKO ${ }^{1}$ is shown in Fig. 3 . Eight receiving dipole antennas are placed on a circle $45^{\circ}$

\footnotetext{
1 www.feko.info
} 
apart from each other. A vertical metal shield is placed between adjacent antennas to reduce the effect of RF signal reverberation. The operating frequency of the RFID system used in the current work is $2.4 \mathrm{GHz}$. The performance metric adopted is the robot's position error $e_{k}$ which is defined as $e_{k}=\sqrt{x_{e}^{2}+y_{e}^{2}}$, where $\left(x_{e}, y_{e}\right)$ is the error in the robot's local coordinate system.
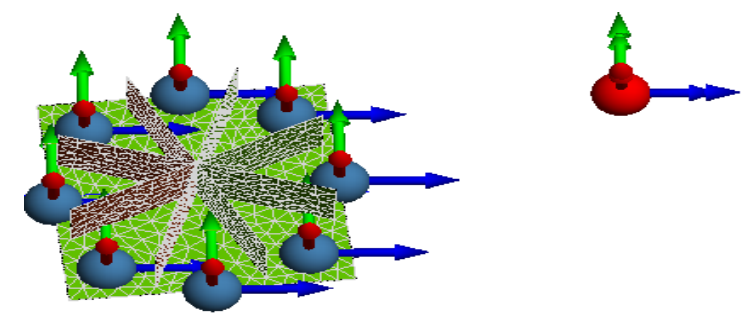

Fig. 3. Antenna system design for an RFID reader using FEKO.

The first test bed was adopted by placing three RFID tags with IDs, 1,2, and 3 , at the 3-D positions $(2,2.5,3) \mathrm{m},(2,4,3) \mathrm{m}$, and $(4,6,3) \mathrm{m}$, respectively. Hence, the target points where the robot is supposed to reach are the 2-D points $\mathrm{p}_{1}=(2,2.5) \mathrm{m}$, $\mathrm{p}_{2}=(2,4) \mathrm{m}$, and $\mathrm{p}_{3}=(4,6) \mathrm{m}$ on the ground, respectively. Fig. 4(a) represents the actual and desired trajectories of the mobile robot during navigation. Initially, the robot is placed at $(4,1) \mathrm{m}$ with an initial orientation of $90^{\circ}$. It then updates its position and orientation based on the RSS measurements of tag 1 to reach point $\mathrm{p}_{1}$. The RSS values during navigation are shown in Fig. 4(b) As can been seen, antenna 3 provides the maximum RSS value once the robot's heading is aligned with the target IDs, 1,2 , and 3 , respectively. This is because antenna 3 is placed at the front castor position of the robot. As the robot moves towards target points, $\mathrm{p}_{2}$ and $\mathrm{p}_{3}$, its corresponding position errors and actual orientations are recorded as shown in Figs. 5(a) and 5(b), respectively. The superiority of applying UKF is clearly observed from the figures in reducing both position and orientation errors. Notice that the position error is enveloped between $\approx 3$ and $15 \mathrm{~cm}$. As can be seen from Fig. 5(b), the robot's current orientation is highly fluctuating when no filtering technique is applied. The smooth updated orientations, however, can be achieved by using UKF, as expected. The percentile error in Fig. 5(a) reveals that during $98 \%$ of the total simulation time, the robot's position error is less than $8 \mathrm{~cm}$ when UKF is used whereas it is $15 \mathrm{~cm}$ or less when it is not.

To better illustrate the performance of the proposed navigation system, six tags were placed in the environment representing the target points $\mathrm{p}_{1}, \mathrm{p}_{2}, \mathrm{p}_{3}, \mathrm{p}_{4}, \mathrm{p}_{5}$, and $\mathrm{p}_{6}$, as shown in Fig. 6(a) The corresponding position errors are presented in Fig. 7(a), The performance of the current navigation system is not affected much by choosing the relative positions of the tags in the sense that the robot could still reach the target points with a satisfactory position error. However, as expected, the orientation errors are higher at the sharp corners of the path which is natural. This is clear from Fig. 7(b)] The RSS values provided by the receiving antennas are presented in Fig. 6(b) which ensures that the front antenna (antenna 3) always provides the maximum RSS values when the 


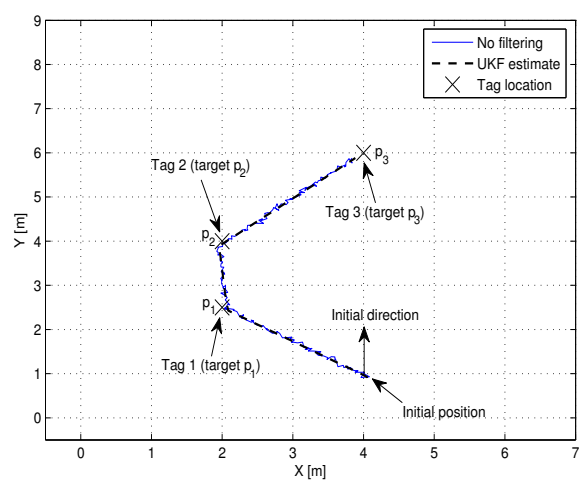

(a)

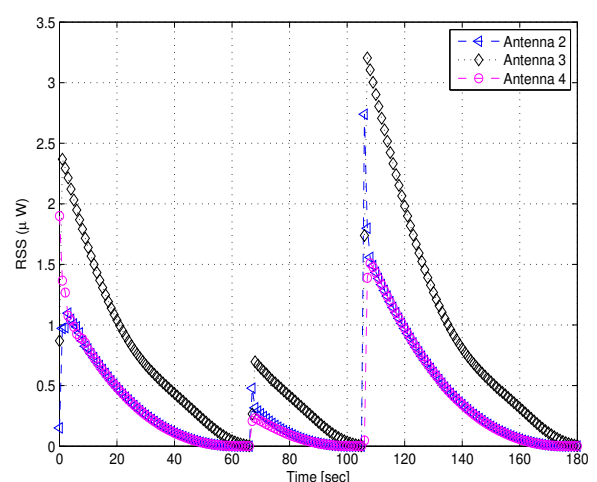

(b)

Fig. 4. (a) Robot's actual vs. desired trajectory for a path with three target points, and (b) RSS measurements received during navigation.

robot's front castor is aligned with the target tag as desired. As for the percentile error in Fig. 7(a) 100\% of the simulation time the position error is less than or close to $12 \mathrm{~cm}$. However, without the UKF position estimation technique, the position error reaches up to $18 \mathrm{~cm}$. This clearly demonstrates the benefit of using UKF for the proposed navigation technique.

\section{Conclusion}

In this paper, we proposed a novel RSS-based mobile robot navigation system using a customized RFID reader which opens up a possibility of applying it in an indoor environment. The RSS measurements are employed as key observations since they can be easily obtained without specialized hardware or extensive training. The robot uses RSS measurement to update its position and orientation as it approach a current target point in the workspace. The positions and orientations are then rectified through UKF. The customized RFID reader is simulated using the electromagnetic software, FEKO. The performance of the proposed system is well demonstrated through computer simulations that reflect the validity and suitability of the proposed navigation system to provide high-performance level in terms of navigation accuracy and scalability.

The proposed navigation system has three major advantages that make it good candidate in many real world indoor navigation systems. Firstly, it is independent of the environment where the robot is deployed. Secondly, the odometry information is not absolutely necessary since it accumulates position and orientation errors over time. Thirdly, an accurate mapping of RSS information to distance is not required as it is the case for most RFID based localization system.

\section{References}

1. K. Finkenzeller, RFID Handbook, 2nd ed. Swadlincote, UK: Wiley, 2004. 


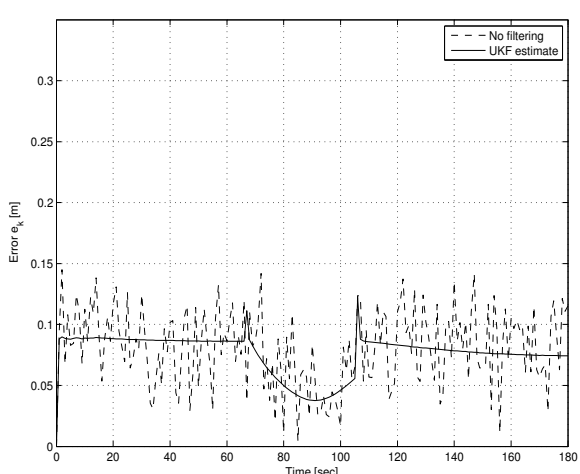

(a)

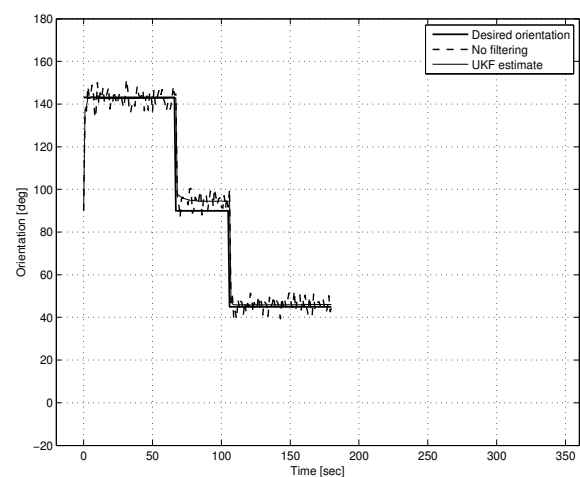

(b)

Fig. 5. Navigation system's performance for a path with three target points, (a) position error, and (b) desired vs. actual orientations.

2. A. Bekkali and M. Matsumoto, "RFID indoor tracking system based on inter-tags distance measurements," in Wireless Technology, ser. Lecture Notes in Electrical Engineering, vol. 44. Springer US, 2009, pp. 41-62.

3. M. Youssef, "The Horus WLAN location determination system," Ph.D. dissertation, University of Maryland, Maryland, 2004.

4. M. Kim and N. Y. Chong, "Direction sensing RFID reader for mobile robot navigation," IEEE Transactions on Automation Science and Engineering, vol. 6, no. 1, pp. 44-54, Jan. 2009.

5. V. Kulyukin, C. Gharpure, J. Nicholson, and S. Pavithran, "RFID in robot-assisted indoor navigation for the visually impaired," in 2004 IEEE/RSJ IROS, Sendai, Japan, 2004, pp. 1979-84.

6. D. Hahnel, W. Burgard, D. Fox, K. Fishkin, and M. Philipose, "Mapping and localization with RFID technology," in Proceedings - IEEE ICRA, no. 1, New Orleans, LA, United States, Apr. 2004, pp. 1015-1020.

7. S. Lanzisera, D. T. Lin, and K. S. J. Pister, "RF time of flight ranging for wireless sensor network localization," in Proceedings of the Fourth Workshop on Intelligent Solutions in Embedded Systems, WISES 2006, Vienna, Austria, Jun. 2006, pp. 165-176.

8. L. M. Ni, Y. Liu, Y. C. Lau, and A. P. Patil, "LANDMARC: indoor location sensing using active RFID," in Proceedings of the First IEEE International Conference on Pervasive Computing and Communications, Fort Worth, TX, USA, 2003, pp. 407-15.

9. S. Miah and W. Gueaieb, "A stochastic approach of mobile robot navigation using customized rfid systems," in International Conference on Signals, Circuits and Systems, Jerba, Tunisia, November 2009.

10. — - "On the implementation of an efficient mobile robot navigation system: An RFID approach," in Proceedings of the International Conference on Intelligent Autonomous Systems (IAS 2010), Ottawa, Canada, August 2010.

11. S. J. Julier and J. K. Uhlmann, "New extension of the Kalman filter to nonlinear systems," in Signal Processing, Sensor Fusion, and Target Recognition VI, vol. 3068, Orlando, FL, USA, 1997, pp. 182-93.

12. S. Thrun, W. Burgard, and D. Fox, Probabilistic Robotics. London, England: The MIT Press, 2005. 


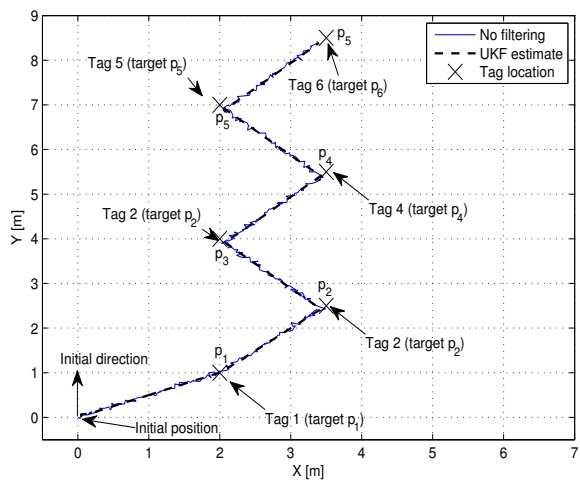

(a)

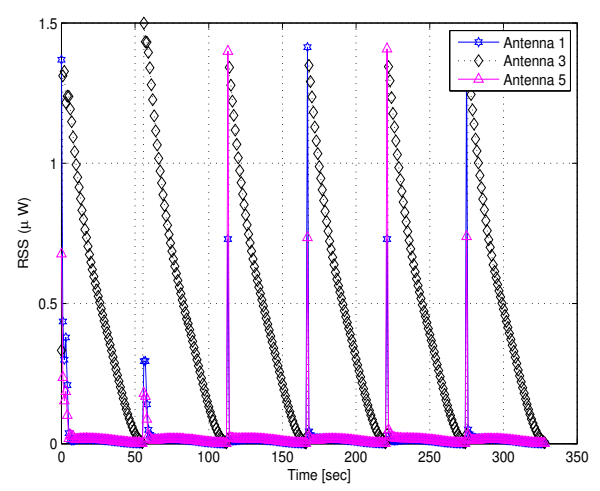

(b)

Fig. 6. (a) Robot's actual vs. desired trajectory for a path with six target points, and (b) RSS measurements received during navigation.

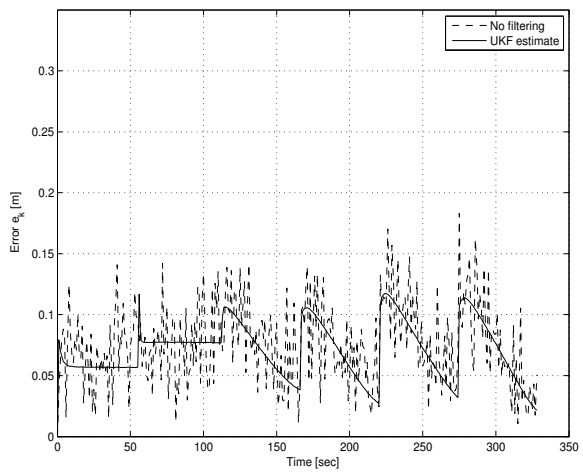

(a)

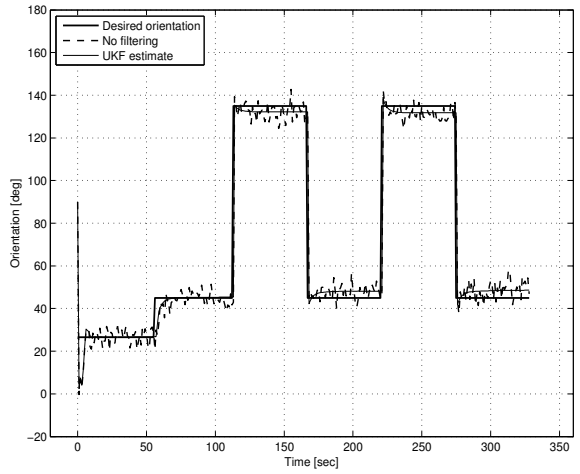

(b)

Fig. 7. Navigation system's performance for a path with six target points, (a) position error, and (b) desired vs. actual orientations. 